\title{
Influence of coherence low intensity optical radiation and radiation periodicity on enzyme activity of embryos of rainbow trout (Oncorhynchus mykiss, Walbaum, I792)
}

\begin{abstract}
Background: The paper presents results of studies of the effect of low-intensity optical radiation on the enzymes activity of rainbow trout embryos under various coherence and periodicity variants. It is shown that polarized radiation of semiconductor laser and the polarized radiation of light-emitting diode have a biological activity. Based on the presented data and also on data obtained previously, we conclude that among the resonant and nonresonant photophysical processes (orientational effect of light, effect of gradient forces, dipole-dipole interactions, thermooptic processes) capable of inducing photobiological effects dependent on such laser-specific characteristics as polarization and coherence, the determining influence in the processes studied in this work comes from the orientational effect of light and dipole-dipole interactions. And the orientational effect can appear for anisotropic media with liquid-crystal type ordering (especially domains in membranes and multiple-enzyme complexes) both under conditions when there is no resonant absorption and for weakly absorbing structures, and can initiate a change in their conformations and accordingly their functional characteristics.
\end{abstract}

Keywords: trout, aquaculture, optical radiation, temperature regimes
Volume 7 Issue 4 - 2018

\author{
MV Shalak,' NV Barulin,' MS Liman, ${ }^{1,3}$ VYu \\ Plavskii \\ 'Department of Ichthyology and Pisciculture, Belarusian State \\ Agricultural Academy, Belarus \\ ${ }^{2}$ BI Stepanov Institute of Physics, National Academy of Sciences \\ of Belarus, Belarus \\ ${ }^{3}$ National Productivity Centre, Nigeria
}

Correspondence: Nikolai Barulin, Department of Ichthyology and Pisciculture, Belarusian State Agricultural Academy, Michurina str. 5, Gorki 213407, Belarus, Tel +375 223370049 , Email barulin@list.ru

Received: June 22, 2018 | Published: July 13, 2018

\section{Introduction}

Reproduction of valuable fish species is a complex technological process, which includes working with breeders, obtaining stocking material, and the formation of a repair and broodstock. In this technological chain, the weakest and most vulnerable link is the production of stocking material due to the high sensitivity of embryos to industrial growing conditions. ${ }^{1}$ During the period of embryonic development in conditions of industrial aquaculture, it is necessary to carry out correction of development using various factors of influence on the body. One of these factors is low-intensity optical radiation, which is successfully used in medicine for treatment, correction and therapy in various aspects. As our long-term studies have shown, laser radiation, as well as the radiation of super-bright LEDs, has a stimulating effect on fish and their sexual products (caviar and sperm), as well as on the development of gullet crustaceans. ${ }^{2,3,4}$ Our previous studies were based on the effect of optical radiation on bioobjects within a single exposure. The question of the most favorable periods of repeated exposure to optical radiation, in which the maximum effect on aquaculture objects is manifested, remains open.

The appearance of lasers available for medical applications has exacerbated the interest not only in studying the therapeutic effectiveness of their radiation, but also in the mechanism of primary photophysical processes that determine the biological activity of this physical factor. The most important and decisive was the question: are the observed effects laser-specific (that is, dependent on the many characteristics of laser radiation, for example, coherence) or are they inherent in any non-laser source of light? The above analysis of the literature data does not allow us to conclude that the data obtained by different authors contradicts in studying the dependence of the biological activity of optical radiation on coherence and polarization, and indicates the need for further research in this direction, with careful control of the characteristics of radiation sources.
One of the most important indicators of metabolism in fish embryos is the activity of enzymes of energy metabolism, lactate dehydrogenase and creatine kinase, which invariably participate in adaptive rearrangements. Measurement of changes in these indicators is widely used in the study of embryonic and postembryonic development of hydrobionts in the studies of many authors. ${ }^{5,6}$

\section{Methods}

The research was carried out at the Department of Ichthyology and Pisciculture and the fish-breeding industrial complex of the Belarusian State Agricultural Academy. As a source of optical radiation, a semiconductor laser (LD) of the phototherapeutic device "Lotos" (red spectral region, wavelength $\lambda=650 \mathrm{~nm}$, coherence length $L_{\text {coh }} 211 \mu \mathrm{m}$ ) was developed in the KB "Lyuzar" and the Physics Institute. B.I. Stepanova National Academy of Sciences of Belarus, as well as a matrix of LED sources of the optical device Strong a (red spectral range $\lambda=630 \mathrm{~nm}$, coherence length $L_{\text {coh }} 26 \mu \mathrm{m}$ ) developed at the Belarusian State Agricultural Academy and B.I. Stepanov Institute of Physics, National Academy of Sciences of Belarus, Belarus. Impact on embryos was carried out for 5 days $(0,24,48,72$ and 96 hours from the start of the experiment) for 20 minutes per day, with a power density of optical radiation of $3.0 \mathrm{~mW} / \mathrm{cm}^{2}$. After exposure of the embryos to optical radiation, they were immediately returned to the refrigerator. In the groups studied, water was replaced daily. The source of water was an artesian well. The water preliminarily underwent deionization, disinfection (UV-irradiated) and temperature equalization. Determination of the activity of enzymes of embryos of rainbow trout was carried out in centrifuged homogenates prepared from whole eggs according to the methods described. The resulting homogenate was used to determine the activity of lactate dehydrogenase (LDH) and creatine kinase $(\mathrm{CK})$. The procedure for determining $\mathrm{LDH}$ and $\mathrm{CK}$ activity is based on the kinetic method recommended by the German Society of Clinical Chemistry (DGKC) ${ }^{7,8}$ which involves measuring 
the rate of change in the absorption coefficient, which is directly proportional to $\mathrm{LDH}$ and $\mathrm{CK}$ activity. $\mathrm{LDH}$ and $\mathrm{CK}$ activity was determined at a temperature of $25^{\circ} \mathrm{C}$ and a wavelength of $\lambda=340 \mathrm{~nm}$.

\section{Results}

Data on the effect of the degree of coherence of polarized optical radiation on the activity of enzymes of embryos of rainbow trout are presented in Figure 1.
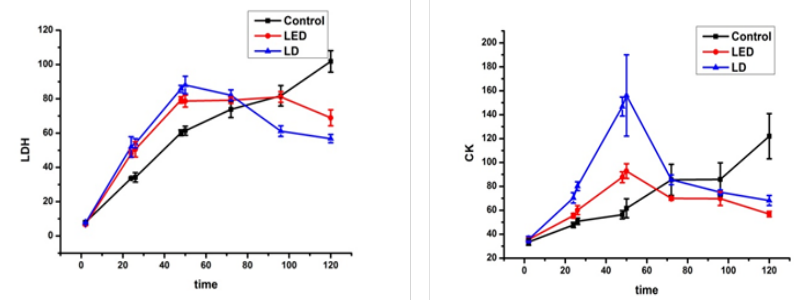

Figure I Changes in the activity of lactate dehydrogenase (LDH) (a) and creatinine kinase (CK) (b) in embryos of rainbow trout under the influence of low-intensity optical radiation of different coherence and periodicity of exposure. (I) is the standard error.

The graphs shown in Figure 1 show the results of comparative experiments when a semiconductor laser (LD) and an LED source are exposed to radiation. From the data presented, it can be seen that, under the influence of LED and LD, a change in the activity of enzymes in embryos of rainbow trout relative to the control group occurred. LDH activity in the two experimental groups significantly differed $(\mathrm{p}<0.05)$ from the control group, at registration of activity after $24,26,48,50$ and 120 hours from the moment of the beginning of the experiment. The activity of $\mathrm{CK}$ in the two experimental groups was significantly different $(\mathrm{p}<0.05)$ from the control group, with activity recorded after 26,48 , and 50hours from the start of the experiment. It should be noted that significant differences between the two different radiation sources were detected only when the LDH activity was recorded 96hours after the start of the experiment and when the $\mathrm{CK}$ activity was registered at 26 and 48hours after the start of the experiment. In all other variants of the comparison, there were no significant differences between the groups studied. As can be seen from the data presented in Figure 1, the activity of LDH and CK in the control group increases, which can be explained by the intensive processes of energy metabolism in the final stage of embryonic development.

\section{Discussion}

The results obtained in this study indicate a pronounced biological activity of low-intensity radiation in the red region of the spectrum, which consists in the ability of light to influence the embryonic development of salmonids. Experimentally this manifests itself in an increase in the activity of enzymes. Effects induced by optical radiation are characterized by a high level of reliability. Comparison of the results of studies carried out using radiation of varying degrees of temporal coherence has shown that the biological effects (enzyme activity) induced by the polarized radiation of a semiconductor laser $\left(\lambda=650 \mathrm{~nm}, L_{\mathrm{coh}} \sim 211 \mu \mathrm{m}\right)$ and an LED source $\left(\lambda=630 \mathrm{~nm}, L_{\mathrm{coh}} \sim 26 \mu \mathrm{m}\right)$. Sources practically do not differ (when studying LDH activity) and differed insignificantly (in the study of CK activity).

The principal conclusion that follows from the presented data is that both polarized radiation of a semiconductor laser and the radiation of a polarized LED source has a biological activity, the degree of temporal coherence is almost 10times smaller.
Based on the presented data and also on data obtained previously $y^{2,3,4}$ we conclude that among the resonant and nonresonant photophysical processes (orientational effect of light, effect of gradient forces, dipoledipole interactions, thermooptic processes) capable of inducing photo biological effects dependent on such laser-specific characteristics as polarization and coherence, the determining influence in the processes studied in this work comes from the orientational effect of light and dipole-dipole interactions. And the orientational effect can appear for anisotropic media with liquid-crystal type ordering (especially domains in membranes and multiple-enzyme complexes) both under conditions when there is no resonant absorption and for weakly absorbing structures, and can initiate a change in their conformations and accordingly their functional characteristics.

\section{Conclusion}

The results obtained in the present work indicate that both polarized radiation of a semiconductor laser and the radiation of a polarized LED source possess a biological activity, the degree of temporal coherence is almost 10 times smaller. It is assumed that the primary photophysical mechanisms that ensure the effect of radiation on metabolic processes in the body are due to cooperative structural transitions in membranes and multi-enzyme complexes due to the orientational action of polarized radiation, as well as dipole-dipole non resonance interactions. The results obtained in the present work also indicate the ability of the periodicity of optical radiation to exert both a stimulating and a depressing effect. The results obtained in the present work, as well as our previous studies; indicate the ability of low-intensity optical radiation to stimulate the embryonic and postembryonic development of aquaculture objects, thereby increasing the efficiency of artificial reproduction technology for valuable fish species.

\section{Acknowledgments}

None.

\section{Conflict of interests}

Author declares that there is no conflict of interest.

\section{References}

1. Kostousov VG, Barulin NV. Development of industrial fish culture in Belarus. Recirculation technologies in indoor and outdoor systems. $H A N D B O O K$.-Research Institute for Fisheries, Aquaculture and Irrigation-Szarvas. 2013:44-48.

2. Plavskii VY, Barulin NV. Effect of polarization and coherence of low-intensity optical radiation on fish embryos. Journal of Applied Spectroscopy. 2008;75(6):843-856

3. Plavskii VY, Barulin NV. Modulation effect of low-intensity laser radiation on its biological activity. Laser Medicine. 2009; 13(1):4-9.

4. Plavskiia VY, Barulin NV. Effect of exposure of sturgeon roe to lowintensity laser radiation on the hardiness of juvenile sturgeon. Journal of Applied Spectroscopy. 2008;75(2):241-250.

5. Ozernyuk ND. Adaptive specific features of energy metabolism in fish ontogenesis. Russian Journal of Developmental Biology. 2011;42(3):201-205

6. Haagensen L, Jensen DH, Gesser H. Dependence of myosin-ATPase on structure bound creatine kinase in cardiac myfibrils from rainbow trout and freshwater turtle. Comp Biochem Physiol A Mol Integr Physiol. 2008;150(4):404-409. 
7. Deutsche Fur Klinische Chemie (DGKC). J Clin Chem Clin Biochem. 1972;10:281-291
8. Deutsche Fur Klinische Chemie (DGKC). J Clin Chem Clin Biochem. $1977 ; 15,255$. 